\title{
STUDY THE PERFORMANCE OF THE TWO WAVELET-BASED ADAPTATION SCHEMES FOR THE SHALLOW WATER FLOW MODELLING
}

\author{
Dilshad Abdul Jabbar Haleem ${ }^{*}$, George Kesserwani ${ }^{* *}$ Aza Hani Shukri ${ }^{*}$ and Alan Saheen Saifaldeen * \\ ${ }^{*}$ College of Engineering, University of Duhok, Kurdistan-Iraq \\ *** Department of Civil Engineering, University of Sheffield, Sheffield-UK
}

\begin{abstract}
This work proposed a new adaptive method which avails from the wavelets theory for transforming the local single resolution information into multiresolution information. This information became accessible and by deactivating or activating them, the spatial resolution adaptation was achieved. The adaptive technique was combined with two standard numerical modelling schemes (i.e. finite volume and discontinuous Galerkin schemes) to produce two new adaptive schemes for modelling one dimensional shallow water flows so-called the Haar wavelets finite volume (HWFV) and multiwavelet discontinuous Galerkin (MWDG) schemes. Both adaptive schemes were tested using hydraulic test cases. The results demonstrated that the proposed adaptive technique could serve as the foundation on which to construct complete adaptive schemes for simulating the real problems of shallow water flow.
\end{abstract}

KEYWORDS: Finite volume method, Discontinues Galerkin method, Haar wavelets, multiresolution onedimensional (1D), shallow water modelling

\section{INTRODUCTION}

$\mathbf{I}$ n the computational hydraulic, the Godunov-type framework and its extension discontiunus Galerkin method have been extensively used in modelling of the shallow water flows due to its properties such as locality, numerical mass conservation and its ability to accommodate the transient complex flow within the numerical solution (see e.g. [1-3]).

The original Godunov-type method was introduced by Godunov in (1959) for the gas dynamic equations by presuming the representation of the numerical solution over each control volume within the discrete domain is piecewise constant and this makes the scheme well suited for solving the problems containing the discontinuities [4]. Later further theoretical and numerical considerations have been given to use the method for simulation the realistic shallow water flow problems such as involving the source term (i.e. the topography term) into the numerical solution and evolving wet/dry region within the computational domain (see e.g. [1, 3, 5-10]). Consequently it is widely applied to real-scale flood simulation and has been integrated into industrial hydraulic modelling software packages. However, large real scale shallow flows present a wide range of spatial scales make the scheme to use dense mesh to obtain accurate results for which mesh adaptation is an optimal solution. Furthermore if the numerical solution of the flowfield contains the complex flow such as shocks or contact discontinuities. The whole discretization domain should be refined uniformly into a large numbers of cells because the position of the complex flows are usually unknown and it is difficult to capture all small scales of shocks waves with the coarse mesh discretization. On the other hand the refinement of the entire domain during the computational flow is trivial task due to the extraordinary computational power required. Therefore the Godunov-type method requires performing mesh adaptation without losing its features. Recently, various adaptive techniques based on domain or solution adaptivity within the context art of finite volume method and its extension to higher order which is called discontinues Galerkin method for modelling shallow water equations, (SWE) have been developed. They include moving mesh methods (see, e.g. [11]) or static grid with locally refinement methods (see, e.g. [12]). These techniques rely on the a priori or posterior knowledge of the numerical solution to produce an adaptive mesh and this leads to introduce uncertainties into the numerical solution [13-14] and to be compromised an error-sensor is designed 
within the numerical scheme. However, an accurate mathematical approach is not available yet to determine an accurate error-sensor for the system of SWE [13]. To avoid using these types of error-estimator sensors the theory of discretewavelets theory incorporates with the context of finite volume method and its extension to higher order which is called discontinues Galerkin method are used for solving the system of conservation law (see e.g.[15-16]). The method takes an advantage of the compression capability of wavelets and dynamically evolves the sparse representation of the locally-structured solution via certain thresholding techniques $[15,17]$. However, the implementation of this idea in solving the system of SWEs preserving the stationary solution (well-balanced) and the evolution of the wet/dry fronts water wave over the topography are not reflected in the literatures apart of recent paper works see [23, 24]. In this work, the Godunov-type finite volume framework and its extension are reformulated by introducing the Haar wavelet bases and Multiwavelets (after scaling and translating) respectively into the framework of the method, in order to obtain an adaptive multiresolution scheme. Here the solution adaptivity can be achieved by omitting the detail coefficients over each cell in which its value falls below a certain prescribed threshold value [13]. The performances of the new adaptive schemes are verified in terms of introducing the friction source term in the shallow water equations and wetting and drying over irregular bed conditions. The paper is organized as follow: Section 2 presents the brief overview of the shallow water model in $1 D$ and section 3 we recall the framework of Godunov-type framework and its extension. In section 4 , we introduce the Haar wavelets and multiwavelets bases and their key features. Section 5 demonstrates how the details and its scaling basis can be incorporated into the Godunov-type method. Section 6 we show several numerical test cases that support our discussion. We close in section 7 with some conclusions and perspectives for future works.

\section{Shallow water equations (SWE)}

In the one dimensional (1D) shallow water flow considering the mass and momentum principle including the topography of the bed, the mathematical model can be cast in a conservative matrix form:

$$
\begin{gathered}
\partial_{t} \mathbf{U}+\partial_{x} F(\mathbf{U})=\mathbf{S} \\
\mathbf{U}=\left[\begin{array}{l}
h \\
q
\end{array}\right], F(\mathbf{U})=\left[\begin{array}{c}
q \\
\frac{g h^{2}}{2}+\frac{q^{2}}{h}
\end{array}\right], \mathbf{S}=\left[\begin{array}{c}
0 \\
-g h \nabla z
\end{array}\right]
\end{gathered}
$$

$t=$ time (sec), $x=$ space $(\mathrm{m})$ and $\mathbf{U}, F(\mathbf{U})$ and $\mathrm{s}$ are vectors containing the conserved variables, the flux and the sources term respectively, in which $h$ is the water depth $(m), q$ is the flow rates per unit width $\left(\mathrm{m}^{2} / \mathrm{s}\right), \mathrm{g}$ is the acceleration gravity $\left(\mathrm{m} / \mathrm{s}^{2}\right.$ ) and $z$ is the bed elevation $(m)$.

\section{Godunov's framework.}

In this section, we present the basic ideas of the Godunov finite volume method. For further details we refer the reader to $[1,5,9]$. The computational

$$
\mathbf{U}_{i}^{t+1}=\mathbf{U}_{i}^{t^{n}}-\frac{\Delta t}{\Delta x_{i}}\left(\mathbf{F}_{i+1 / 2}-\mathbf{F}_{i-1 / 2}\right)+\Delta t \mathbf{S}_{i}
$$

Where $\mathbf{U}_{i}^{n+1}$ represents the computing value of the conserved variables in the next time level and the discrete values $\mathbf{U}_{i}^{t^{n}}$ propose the average approximated numerical solution in the current time level. $\mathbf{F}_{i \pm 1 / 2}$, is the numerical fluxes at cell interfaces and in this work it is based on the Roe's domain of $1 D$ is divided into $N$ uniform and nonoverlapping cells with boundary points $0=x_{1 / 2}<x_{3 / 2}<\ldots .<x_{N+1 / 2}=L$. A cell $i$ is defined as $I_{i}=\left[x_{i-1 / 2}, x_{i+1 / 2}\right]$ with a cell size $\Delta x=x_{i+1 / 2}-x_{i-1 / 2} \quad$ and a centre $x_{i}=\left(x_{i+1 / 2}+x_{i-1 / 2}\right) / 2$. By integrating the equation (2) in space and time $\left[t^{n+1}, t^{n}\right]$ over the $i^{\text {th }}$ cell yields the following discrete conservative form of the SWE:

Riemann solver [18]. The local numerical source flux is $\mathbf{S}_{i}$. Hence the scheme is defined as explicit. The equation (3) can be written in the semidiscrete form with considering the three values of numerical solution $\left(\mathbf{U}_{i-1}, \mathbf{U}_{i}, \mathbf{U}_{i+1}\right)$ such that .

$$
\mathbf{U}_{i}^{t^{n+1}}=\mathbf{U}_{i}^{t^{n}}+\Delta t L\left(\mathbf{U}_{i}\right)
$$




$$
L=-\frac{1}{\Delta x_{i}}\left(\stackrel{R o e}{\mathbf{F}}\left(\mathbf{U}_{i-1}, \mathbf{U}_{i}\right)-\stackrel{R o e}{\mathbf{F}}\left(\mathbf{U}_{i}, \mathbf{U}_{i+1}\right)\right)+\int_{I_{i}} \mathbf{S}\left(\mathbf{U}_{i}\right)
$$

According to the stability condition of the scheme the Courant-Friedrichs- Lewy (CFL) is defined and will be discussed later. The bed discretization technique that has been defined within the Godunov-type method by Bouchut [1] is adopted in this work in order to preserve the stationary solution (well-balanced) of the system (1). Furthermore, the techniques that have been employed to deal with wet/ dry zones over the computational domain are taken from the Auduse's work [10].

\section{Scaling basis function and Haar wavelets}

In this section, we summarize the basic conceptual properties of scaling basis functions, multiresolution analysis, Haar wavelets and the main steps of algorithm of WFV method of employing the local decomposition and

$$
\phi_{j}^{n}(x)=2^{\frac{l}{2}} \phi\left(2^{n}(x+1)-2 j-1\right) \quad, n, j=0,1, \ldots 2^{n}-1 \in Z \quad \phi \in L^{2}(\Re)
$$

Where $n$ refer to the resolution level and the translation factor is $j$. Here we also define a closed span which are scaled and translated version of $\phi_{j}^{n}(x)$

$$
V_{n} \equiv \operatorname{Span}\left\{2^{\frac{l}{2}} \phi_{j}^{n}\left(2^{n} x-j\right)\right\}
$$

If we define two-scale sequence of $\phi$ i.e. $l=0,1$ and substitute them into (6), we will obtain a set of bases functions that allow us to represent any function $f(x) \in L^{2}$ into the subspaces $V_{0}$ and $V_{1}$ respectively.

Since these bases are scaled and shifted within closed span then the bases in $V_{0}$ contained in $V_{1}$ or $V_{0} \subset V_{1}$. This leads to obtain the same shape pattern of represented function in both subspaces with different resolution. This representation leads to generate the concept of nested subspaces which is known the corner stone of wavelet theory. Here the single scale functions are modified to support interval $[-1,1]$ instead of $[0,1]$ see figures $1-4$. Furthermore, their dilation and translation at coarse level $n=0$ generates the nested subspaces of closed space $V_{0}$ and satisfies the following properties which called also the multiresolution properties MRA. reconstruction algorithm of the numerical solution. More details can be found in [17, 19]

The first step of obtaining the wavelets functions is presuming all scaling functions are belonging to the Hilbert space $L^{2}(\Re)$ and they are non-overlapping i.e. orthogonal among themselves. Thus we can span the space of these functions only by applying dilation (scaling) and translation (shifting) processes on the mother function $\phi \in L^{2}(\mathfrak{R})$. This means a function $\phi(x)$ can be written as a linear combination of $\phi(2 x)$, $\phi(2 x-1)$ with respect to the compact support of the mother function. The general form of the refinement scaling function can be written as follow:-

$$
\begin{array}{ll}
\text { 2. } & \bigcap_{l \in Z} V_{i}=\{0\} \\
\text { 3. } & \bigcup_{i \in Z} V_{n}=L^{2}(\Re) \\
\text { 4. } & f(x) \in V_{k} \Leftrightarrow f(2 x) \in V_{k+1}, k=0,1, \ldots, n \in Z
\end{array}
$$

Consider the nested sequences of subspaces $V_{n}$ as in property 1 of MRA. These subspaces are non-overlapping. Thus, we can define the orthogonal complement subspace $W_{n}$ (wavelets space) between any two sequences of subspaces $V_{n-1}$ and $V_{n}$ that is,

$V_{l+n}=V_{n} \oplus W_{n}, \quad n \in Z, \quad V_{n} \perp W_{n}$

Since all these subspaces are orthogonal. Consider the properties 2 and 3 of MRA, this leads to introduce an orthogonal decomposition of Hilbert space $L^{2}(\Re)$.

$L^{2}(\Re)=V_{n} \underset{k=0}{\stackrel{n-k-1}{\oplus}} W_{n+k} \quad$ for $\quad n<k, \quad n, k \in Z$

Furthermore, the $W_{n}$ spaces inherit the MRA property 4 from the $V_{l}$;

$f(x) \in W_{k} \Leftrightarrow f(2 x) \in W_{k+1}, k=0,1, \ldots, n \in Z$

Thus, given any scaling function $\phi \operatorname{in} V_{n}$, there exists another function $\psi$ in $W_{n}$ called wavelets see figure 2 and 3 , such that

$$
\psi_{j}^{n}(x)=2^{\frac{l}{2}} \psi\left(2^{n}(x+1)-2 j-1\right), n, j=0,1, \ldots 2^{n}-1 \in Z
$$




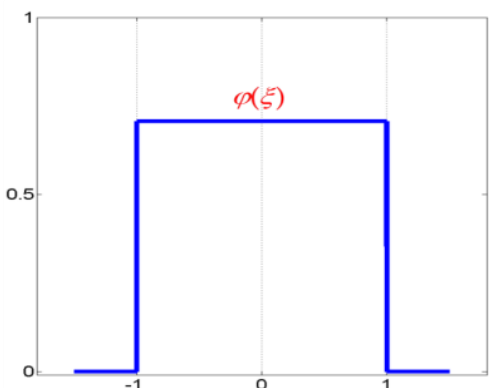

Fig.(1):- The scaling function of Haar wavelets in level $n=0$.

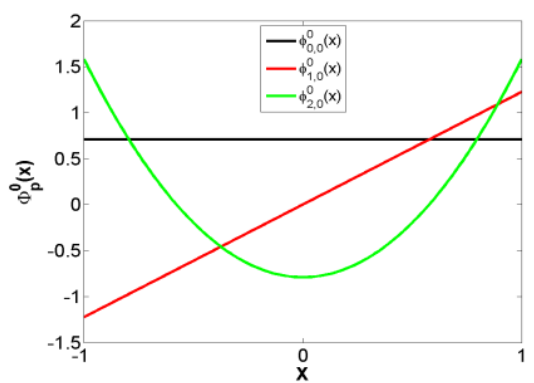

Fig.( 3):- The scaling functions of Multiwavelets in level $n=0 ., p=0,1,2$

The decomposition and reconstructions structure of the local numerical solution across different resolution is obtained via considering the two types of filters: the averaging filter $\left.h_{j}^{n}\right|_{\substack{n=1,2 \\ j=0,1, \ldots,-1}}$ (belong to the scale functions) and the differencing filter $\left.g_{j}^{n}\right|_{\substack{n=1,2 \\ j=0,1}}$ (belong to the wavelets and multiwavelet functions) where $h_{j}^{n}=\left\langle\phi_{j}^{n-1}, \phi_{j}^{n}\right\rangle, g_{j}^{n}=\left\langle\phi_{j}^{n-1}, \psi_{j}^{n}\right\rangle$. The relationship that allows us to downscale the local solution such as from level $n=1$ to the level $n=0$ with corresponded details coefficient $\mathbf{D}_{j}^{n}$ can be define via the average and different filtering as follow:

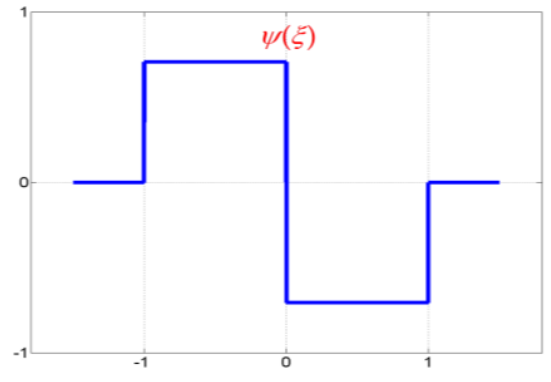

Fig.( 2):- The Haar wavelets in level $n=0$.

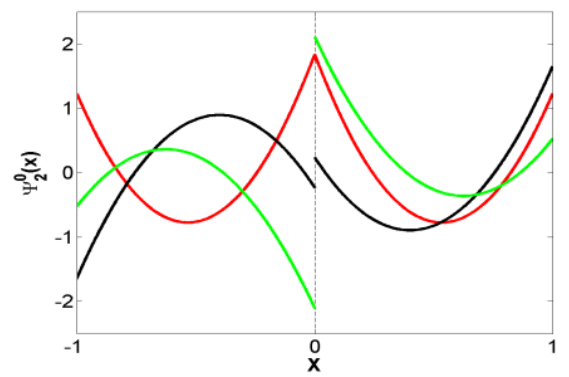

Fig.( 4):- The Multiwavelets in level $n=0$. considering the associated scaling functions

$$
\begin{aligned}
& \mathbf{U}_{i 0}^{0}=\left(h_{0}^{1} \mathbf{U}_{i 0}^{1}+h_{1}^{1} \mathbf{U}_{i 1}^{1}\right) \sqrt{\frac{1}{2}} \\
& \mathbf{D}_{i, 0}^{0}=\left(g_{0}^{1} \mathbf{U}_{i 0}^{1}+g_{1}^{1} \mathbf{U}_{i 1}^{1}\right) \sqrt{\frac{1}{2}}
\end{aligned}
$$

By using the same filters which together with the local solution in level zero and their associated detail coefficients allow us to upscale the local solution from the level zero to the level one as follows:

$$
\begin{aligned}
& \mathbf{U}_{i 0}^{1}=\left(h_{0}^{1} \mathbf{U}_{i 0}^{0}+g_{0}^{1} \mathbf{D}_{i 0}^{0}\right) \sqrt{2} \\
& \mathbf{U}_{i 1}^{1}=\left(h_{1}^{1} \mathbf{U}_{i 0}^{0}+g_{1}^{1} \mathbf{D}_{i 0}^{0}\right) \sqrt{2}
\end{aligned}
$$

By the recursive application of equations (10) to (13) the solution can be promoted to higher resolution level or alternatively demoted to a lower resolution levels (see Figure 5).

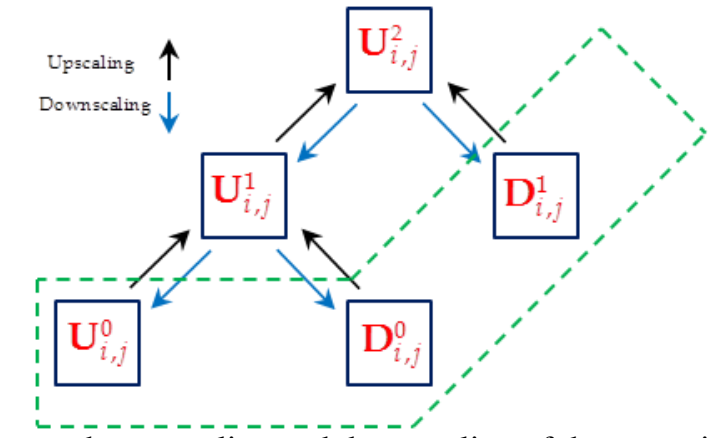

Fig. (5):- Sketch demonstrates the up-scaling and downscaling of the numerical solution across different resolution levels. 


\section{Adaptive Schemes}

In order to obtain adaptive scheme the discrete Haar wavelets, Multiwavelets and their transformation in section 4 are combined with the corresponding non-adaptive schemes in section 3 which yield Haar adaptive finite volume scheme (HWFV) and Multiwavelets discontinuous Galerkin scheme considering second order accuracy (MWDG2) . The combination is straightforward to extend the full discretisation in space and time. The starting point is a given Godunov-type framework discretization is defined on the uniform highest resolution level $(n=2)$. Then performing the downscale of the numerical solution over each cell to obtain all detail coefficients plus the numerical solution in level zero and then apply the thresholding to discard the non-significant details in order to reduce the

$$
\begin{aligned}
& \mathbf{U}_{i, j}^{n_{i, j} t^{n+1}}=\mathbf{U}_{i, j}^{n_{i} t^{n}}+\Delta t L\left(\mathbf{U}_{i, j}^{n i}\right) \\
& L_{i j}^{n_{i}}=-\frac{2^{n_{i}}}{\Delta x_{i}}\left(\stackrel{R o e}{\mathbf{F}}\left(\mathbf{U}_{i-1, j}^{n_{i}}, \mathbf{U}_{i j}^{n_{i}}\right)-\stackrel{\text { Roe }}{\mathbf{F}}\left(\mathbf{U}_{i j}^{n_{i}}, \mathbf{U}_{i+1 j}^{n_{i+1}}\right)\right)+\int_{I_{i j}^{n_{i}}} \mathbf{S}\left(\mathbf{U}_{i j}^{n_{i}}\right)
\end{aligned}
$$

Here the index $j$ is associated with the resolution level of the local cell.

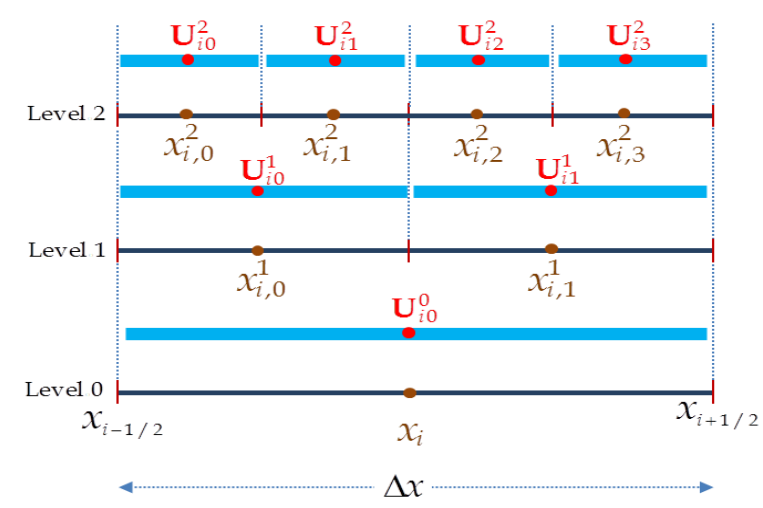

Fig.(6):- The Multiple resolution levels over the reference cell $I_{i, j}^{n}$ with associated local numerical solution.

\subsection{CFL Criterion}

The current wavelet- based finite volume scheme is overall explicit and its numerical stability is controlled by imposing the CourantFriedrichs- Lewy (CFL) criterion. The suitable time step $\Delta t$ can be obtained by the following formula:

$$
\Delta t=C \cdot \min \left[\frac{2^{-n} \Delta x}{\left|u_{i, j}^{n}\right|+\sqrt{g h_{i, j}^{n}}}\right]
$$

Where $\Delta x$ the dimension of the cell at is coarse level; $u_{i, j}^{n}$ is the depth-averaged velocity at the centre of the cell or sub-cell which depends to the local resolution level of the cell. $\sqrt{g h_{i, j}^{n}}$ is the wave celerity and $C$ is the Courant number $(0 \leq C \leq 1)$, in the computation, it is normally set to be 0.98 [20].

\subsection{Thresholding}

Recall in Haar wavelets the filter $H$ is an averaging filter while its mirror counterpart $G$ produces details. The wavelets coefficients correspond to details. When the details are small 
i.e. the numerical solution is smooth, they could be cancelled without substantially affected to the accuracy of solution. Therefore, the idea of thresholding the details coefficients is a way of reducing the density of the adaptive cells over the computational domain. However, in the real computations, it is impossible to compute the exact value of it because it is test case depended $[13,21]$. Here, the threshold value at coarse level is $\varepsilon=0.01$ and it is normalized in correspond with the resolution level.

\subsection{Prediction step}

In WFV scheme, it is important after each time step to predict the local resolution level over each cell, in order to guarantee that no significant (future) feature of the numerical solution is omitted. It is only based on the information available at the current level and it is associated with numerical solution that requires to be updated by the WFV scheme. The following steps requires throughout its employed

Step (i). Find the scale coefficients of the conserved variables at level $\mathrm{n}=0$.

Step (ii). The normalized gradient $(\alpha)$ between the local cell and its neighbor cell was calculated by using this formula:

$$
\alpha=\frac{\left|\mathbf{U}_{i, 0}^{0}-\mathbf{U}_{i+1,0}^{0}\right|}{\max \left(1,\left|\mathbf{U}_{i, 0}^{0}\right|\right)}
$$
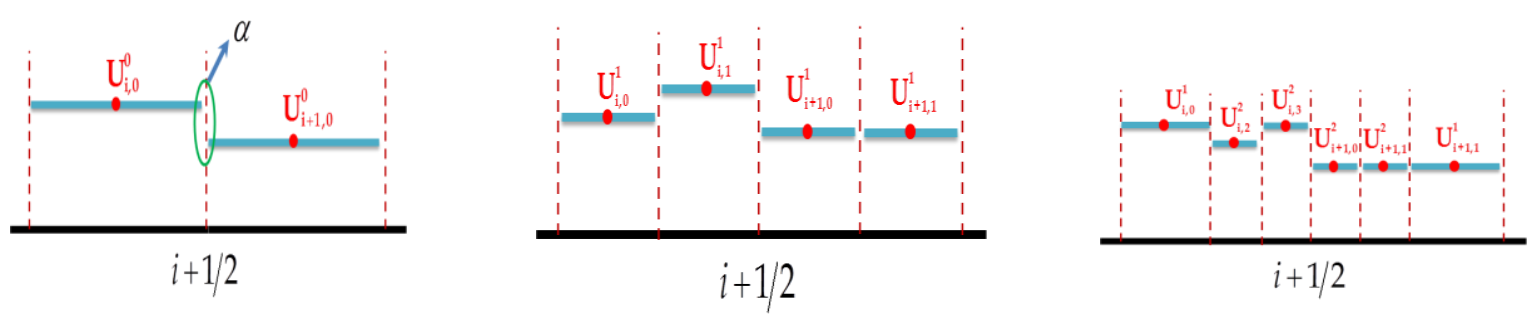

Fig.( 7):- Illustrates the spatial resolution level due to the prediction step between cells $i$ and $i+1$ : the upper row $(\alpha \geq 0.1)$, the middle row $(0.1>\alpha \geq 0.05)$, the lower row $(0.05>\alpha)$.

\section{Numerical results \\ 6.1 Steady hydraulic jump with friction in a rectangular channel}

The purpose of this test case is to illustrate the behaviour of both schemes in performing the adaptivity process considering the bed-friction with the source term. This test case is based on the set-up proposed by MacDonald [25]. A hydraulic jump is assumed to be formed in a prismatic rectangular channel which has $1000 \mathrm{~m}$ long and 20 $m$ width. The Manning's roughness coefficient is set to 0.02 and the bed slope is spatially varied.
Step (iii). The resolution mesh between the local cell and its neighbor cell has the following forms. If $(\alpha \geq 0.1)$ the resolution mesh would be as illustrated in the upper row of figure 7 . Whereas the form of mesh would be the same as in the middle row of figure 7 if $(0.1>\alpha \geq 0.05)$ and if ( $0.05>\alpha$ ) the mesh has the form which is illustrated in the lower row of Figure 4.

Here, the values of 0.1 and 0.05 is only used as indicators for deciding the mesh forms and these are not affected to the numerical solution of the predicted cells because in this scheme the algorithms of transformation from the low resolution level to the high resolution level for those cells need only the zeros detail coefficients. Furthermore, that algorithm was utilized by Harten [15]. With concerned the significant details coefficients in the context of finite volume for numerical solution of the hyperbolic system and this turned out to give the satisfactory results. It is convenient to introduce the two indicators to decide the resolution levels of the active cells neighbors without destroy the numerical solution and that is necessary to capture all features of the propagation of water wave during the computational time. 


\subsubsection{HWFV solution}

The baseline mesh has 30 cells, which results 120 cells at the highest level (i.e. $n=2$ ). The nonadaptive computation is performed using the same number of cells that the adaptive solution is needed to converge the steady hydraulic jump. The convergence of the adaptive solution is achieved with 46 cells. This is performed for the purpose of comparison.

The simulations are noted to converge at around $\mathrm{T}=600 \mathrm{~s}$. Figure 8 shows the results of the adaptive and uniform conditions, together with the exact solution. Generally, the numerical solutions match together with the analytical solution and they overlap almost fully. However, some differences exist, particularly in the flow rate solutions, see Figure 8.b, and that due to the chosen discretisation of the friction source term, as previously reported by Burguete Tolosa et.al.[26] Clearly, the adaptive solution has more cells for capturing the hydraulic jump compared to the uniform schemes solution and, at the subcritical region, the uniform solution is slightly less accurate, see Figure 8.a. Based on this, the adaptive scheme can refine more cells through promoting the local solution $(n=2$ at the hydraulic jump region and $n=1$ at the subcritical region, see Figure 8.c), to minimize errors at the same computational cost.

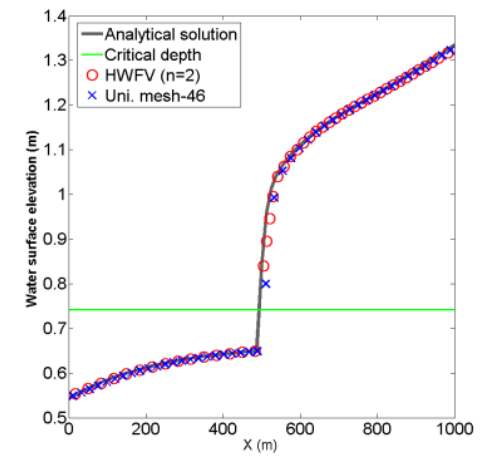

a. water surface elevation

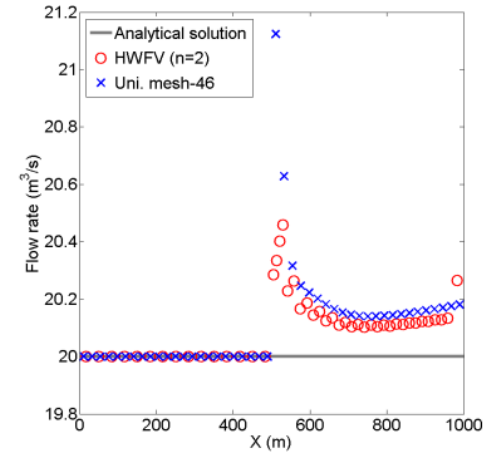

b. Flow rate

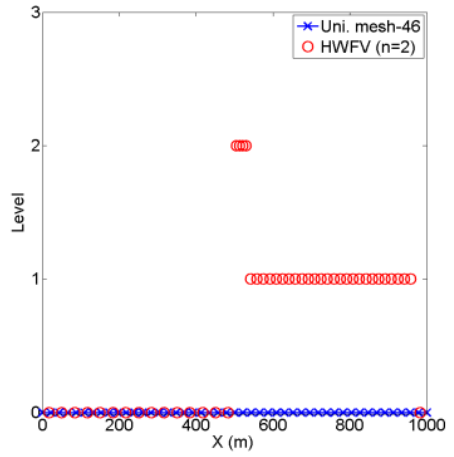

c. Level

Fig.( 8):- The results of HWFV scheme for Steady hydraulic jump in a prismatic rectangular channel

\subsubsection{MWDG2 solution}

The domain of the computation at $n=0$ is divided into 15 cells and the adaptive computation is performed with $n=2$ levels, which results in 60 cells. This discretization of the domain provides 120 degrees of freedom. Thus, the performance of the comparisons between the adaptive results obtained from both schemes (i.e., HWFV and MWDG2) becomes reasonable. The adaptive solution converges with a maximum of 25 cells and this number of cells is used to run the nonadaptive computation. This is performed to compare its results with the adaptive solution results. The convergences of the solutions are achieved at around $\mathrm{T}=530 \mathrm{~s}$ which is less compared with the HWFV scheme and this is due to the advantage of the DG2 over FV schemes. Figure 9 presents the results for both the adaptive and uniform conditions, together with the analytical solution. The numerical solutions of both schemes have a good agreement with the analytical solution and they generally overlap throughout the domain with some difference in terms of the distribution of cells in the domain, see Figure 9.a. At the hydraulic jump and subcritical regions the adaptive scheme has refined more cells in contrast with the supercritical region, in which the adaptive scheme retains coarse level. This implies that the MWDG2 scheme responses to the flow conditions in an optimal way compared to DG2 scheme. It is clear that the adaptive MWDG2 scheme has transferred successfully the feature of the standard DG2 of preserving the constant discharge. In addition, the adaptive scheme also offers more cells where the hydraulic jump occurs which leads to capturing it better when compared to the uniform numerical solution results. Furthermore, at the smooth region, particularly in the supercritical flow region, the MWDG2 scheme allows for coarser cells which result in a reduction of computational cost when compared to the uniform computation. 


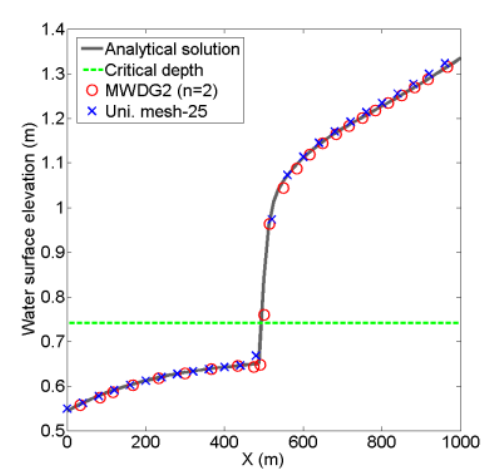

a. water surface elevation

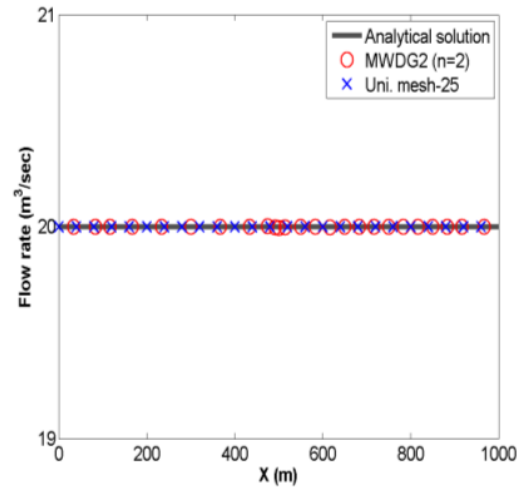

b. Flow rate

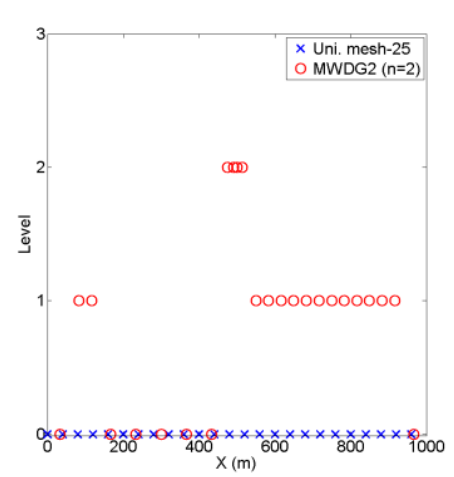

c. Level

Fig. (9):- The results of MWDG2 scheme for Steady hydraulic jump in a prismatic rectangular channel

\subsubsection{Comparisons between HWFV and MWDG2 Schemes}

In comparing of the two schemes, the MWDG2 scheme is generally better in capturing the steady discharge solution throughout the domain. The both schemes have an advantage over the uniform scheme (i.e., FV and DG2) in terms of responding to the flow condition feature, which they refine more cells where the hydraulic jump occurs. Irrespective of the baseline mesh size, the HWFV and the MWDG2 schemes require around $38 \%$ and $42 \%$ respectively, less in computational efforts, than the simulation on the associated fine uniform mesh. These percentages are reasonable because their degrees of freedom are set to be same at $\mathrm{t}=0 \mathrm{~s}$. However, these percentages change with time. In Figure 10, which shows the evolution of the number of active cells and their variance with $\mathrm{N}_{2}$ ? At around $\mathrm{t}=80 \mathrm{~s}$, too many cells are being activated by the MWDG2 scheme which is around $75 \%$ of the equivalent uniform scheme. While in the HWFV scheme, the adaptive solution requires a maximum of $51 \%$ of the counterpart uniform mesh. The magnitude of $N / \mathrm{N}_{n}$ for both adaptive schemes is relatively the same when $200 s \leq \mathrm{t} \leq 300 s$ and it becomes almost constant with $\mathrm{t} \geq 400 \mathrm{~s}$.

It is clear that efficient adaptive processes are obtained with the selected baseline meshes in both adaptive schemes and also the active cell profiles of both schemes have almost the same response to the flow conditions regardless how many cells are being activated during the simulation. Furthermore, the possibility of coarsening baseline mesh for both adaptive schemes is feasible, since both adaptive schemes are not activating all accessible cells during the simulation.

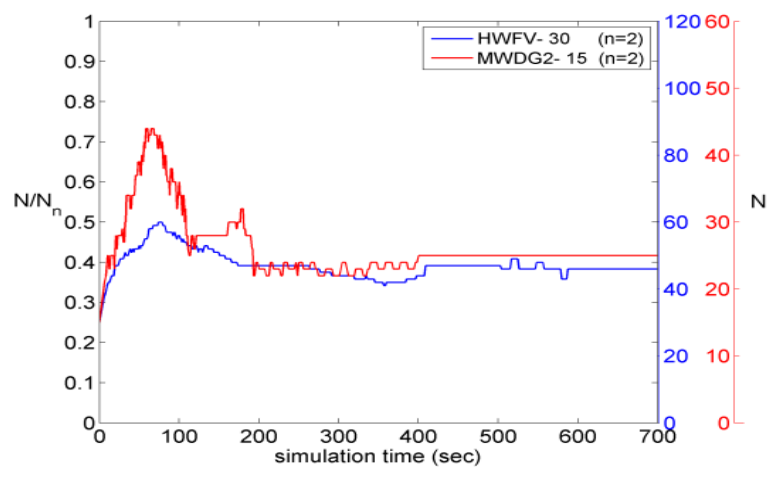

Fig.( 10.):- The active cells evolution of the adaptive schemes for the steady hydraulic jump with friction bed in the rectangular channel.

\subsection{Quiescent flow over an irregular bed}

The quiescent flow (well-balancing) property was introduced first by Bermudez and Vazquez [22] and has been broadly used by numerical scheme developers (see, among others, $[6,10]$ and
[14]). Therefore a test case which takes into consideration a differentiable and nondifferentiable topography, defined by the equation 18 and shown in Figure 11a, is introduced to 
achieve the well-balancing property for both the

$$
z(x)= \begin{cases}0.2\left(0.05(x-10)^{2}\right) & \text { if } 8<x<12 \\ 0.05 x-1.1 & \text { if } 22 \leq x<25 \\ -0.05 x+1.4 & \text { if } 25 \leq x \leq 28 \\ 0.3 & \text { if } 39<x<46 \\ 0 & \text { otherwise }\end{cases}
$$

In addition, the adaptive schemes are devoted to resolve complicated flows over topography through considering three different cases: (i) wet/dry fronts case (i.e. at both sides of the piecewise constant elevation); (ii) critical wet case (i.e. $h=0 \mathrm{~m}$ at the peak of a bump) and (iii) fully wet (i.e. the computational domain is wet apart from regions $(i)$ and $(i i)$ ). The computational domains at the coarse level for the HWFV and MWDG2 schemes are comprised of 50 cells and 25 cells, receptively. The computational models are run up to $1000 s$ with $n=3$. An upstream boundary condition is imposed by zeroing flow rate whereas a downstream boundary condition is set to $0.2 \mathrm{~m}$. These imposed conditions are also used to initialize the simulation. Figures 11 and 12 show the numerical results of the adaptive scheme respectively, considering the full solution of the FV and DG. The resolution level throughout the domain in both adaptive schemes is almost the same in the wet/dry fronts case, see Figures 11.c adaptive schemes.

and 12.c. Whereas at the triangular hump, the HWFV scheme retains the resolution level at 2, but the resolution level is varied between $n=2$ and $n=3$ in the MWDG2 scheme. This is expected to be caused by the extra slope coefficient values that are more sensitive and are therefore activated by the adaptive scheme, see Figure 12.b. The adaptive schemes succeed in capturing; the critical wet region at the peak of the bump, but the MWDG2 scheme refines more cells to the highest resolution level. This is attributed to the involvement of the slope details coefficients in the adaptive solutions. The full adaptive solutions of the free surface elevation preserve the motionless steady state throughout the simulations (see Figures 11.a and 12.a) and, the zero flow rate in both adaptive computations is accurately replicated within the range of machine precision $\left(1 \times 10^{-16}\right)$ (see Figures 11.b and 12.b).

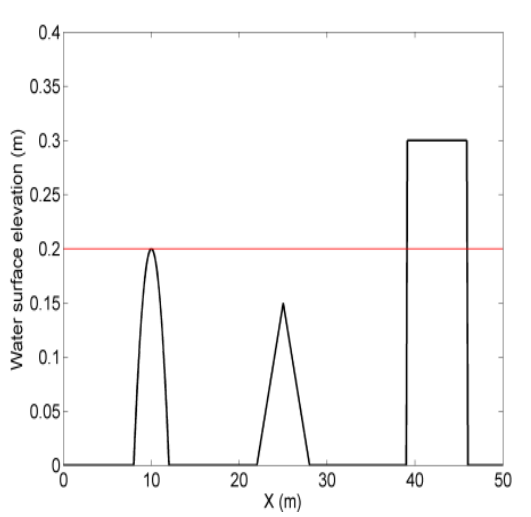

a. water surface elevation

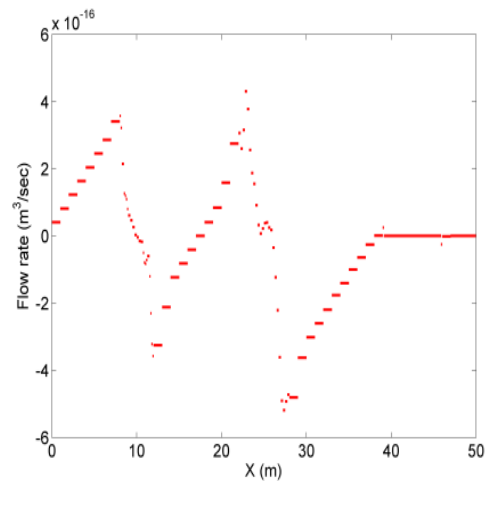

b. flow rate

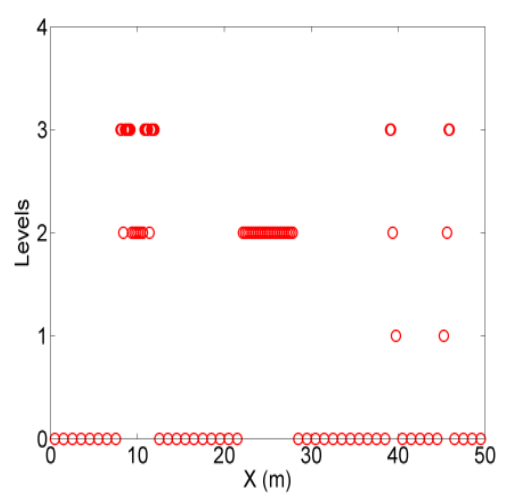

c. level

Fig.( 11.):- Quiescent flow with wet/dry fronts for HWFV scheme 


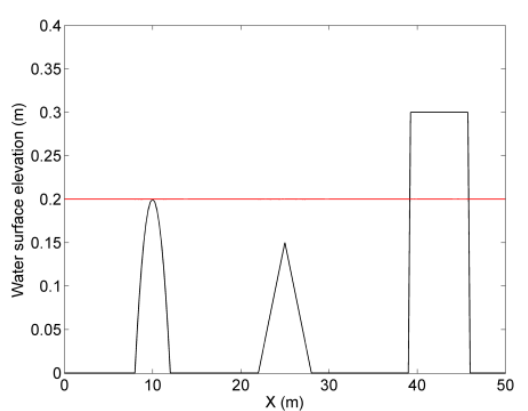

a. water surface elevation

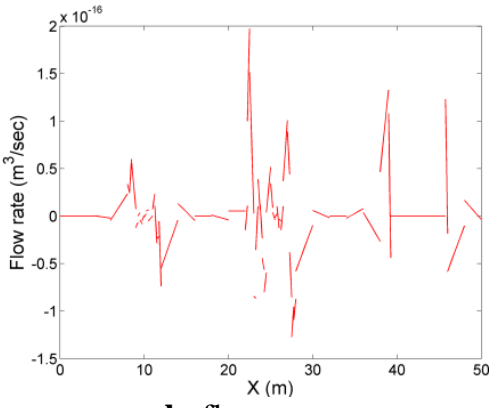

b. flow rate

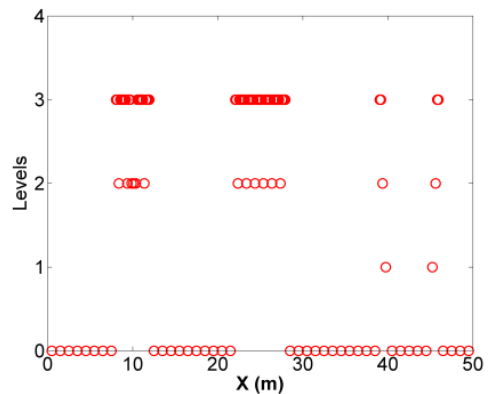

c. level

Fig.( 12.):- Quiescent flow with wet/dry fronts for MWDG 2 scheme

\section{CONCLUSIONS}

In this work, by combining wavelet-based scaling functions with the design of both standard shallow water flow modelling schemes (i.e. FV and DG), two new adaptive schemes (i.e. HWFV and MWDG2) have been obtained. In these new schemes, the spatial resolution adaptivity is achieved from the local solution itself and it is based on a single user-prescribed parameter The adaptive schemes was able to retain all properties from the reference uniform counterpart schemes such as the mass conservation, shock capturing, well-balancing, moving wet/dry fronts and slope limiter properties. This means if any property is valid or improved for the reference uniform schemes; it will be transferable into the adaptive counterpart's schemes. Response of the accuracy to the adaptivity process via fixing the degrees of freedom (DOF) in both adaptive schemes (when $t$ $=0 \mathrm{~s}$ ) was shown a good performance in terms of providing almost the same computational efforts when the adaptive solutions converged. Altogether, the results demonstrated that the multiwavelets adaptive technique has successfully merged into the FV and DG2 frameworks. The results are also encouraging and suggesting that the adaptive schemes can provide a rigorous, robust and efficient solution to the multi-scale problems arise in shallow water modelling.

\section{REFERENCES}

1. Bouchut, F., Efficient numerical finite volume schemes for shallow water models. Edited Series on Advances in Nonlinear Science and Complexity, 2007. 2: p. 189-256.

2. Toro, E.F., Shock-capturing methods for freesurface shallow flows. 2001: Wiley.

3. Toro, E.F. and P. Garcia-Navarro, Godunov-type methods for free-surface shallow flows: A review. Journal of Hydraulic Research, 2007. 45(6): p. 736-751.
4. Harten, A., P.D. Lax, and B.v. Leer, On upstream differencing and Godunov-type schemes for hyperbolic conservation laws. SIAM review, 1983. 25(1): p. 35-61.

5. Fraccarollo, L., H. Capart, and Y. Zech, A Godunov method for the computation of erosional shallow water transients. International journal for Numerical Methods in fluids, 2003. 41(9): p. 951-976.

6. Kesserwani, G., Topography discretization techniques for Godunov-type shallow water numerical models: a comparative study. Journal of Hydraulic Research, 2013. 51(4): p. 351-367.

7. Bradford, S.F. and B.F. Sanders, Finite-volume model for shallow-water flooding of arbitrary topography. Journal of Hydraulic Engineering, 2002. 128(3): p. 289-298.

8. Begnudelli, L. and B.F. Sanders, Conservative wetting and drying methodology for quadrilateral grid finite-volume models. Journal of Hydraulic Engineering, 2007. 133(3): p. 312322.

9. Garcia-Navarro, P. and M.E. Vazquez-Cendon, On numerical treatment of the source terms in the shallow water equations. Computers \& Fluids, 2000. 29(8): p. 951-979.

10. Audusse, E., et al., A fast and stable well-balanced scheme with hydrostatic reconstruction for shallow water flows. SIAM Journal on Scientific Computing, 2004. 25(6): p. 2050-2065.

11. Skoula, Z., A. Borthwick, and C. Moutzouris, Godunov-type solution of the shallow water equations on adaptive unstructured triangular grids. International Journal of Computational Fluid Dynamics, 2006. 20(9): p. 621-636.

12. Nikolos, I. and A. Delis, An unstructured nodecentered finite volume scheme for shallow water flows with wet/dry fronts over complex topography. Computer Methods in Applied Mechanics and Engineering, 2009. 198(47): p. 3723-3750.

13. Hovhannisyan, N., S. Müller, and R. Schäfer, Adaptive multiresolution discontinuous galerkin schemes for conservation laws. Mathematics of Computation, 2014. 83(285): p. 113-151. 
14. Kesserwani, G. and Q. Liang, Dynamically adaptive grid based discontinuous Galerkin shallow water model. Advances in Water Resources, 2012. 37: p. 23-39.

15. Harten, A., Multiresolution algorithms for the numerical solution of hyperbolic conservation laws. Communications on Pure and Applied Mathematics, 1995. 48(12): p. 1305-1342.

16. Müller, S., Adaptive multiscale schemes for conservation laws. Vol. 27. 2003: Springer.

17. Keinert, F., Wavelets and multiwavelets. 2003: CRC Press.

18. Roe, P.L., Approximate Riemann solvers, parameter vectors, and difference schemes. Journal of computational physics, 1981. 43(2): p. 357-372.

19. GUF, J.-S. and W.-S. Jiang, The Haar wavelets operational matrix of integration. International Journal of Systems Science, 1996. 27(7): p. 623628.

20. Cockburn, B. and C.-W. Shu, Runge-Kutta discontinuous Galerkin methods for convectiondominated problems. Journal of scientific computing, 2001. 16(3): p. 173-261.

21. Gerhard, N. and S. Müller, Adaptive multiresolution discontinuous Galerkin schemes for conservation laws: multi-dimensional case.
Computational and Applied Mathematics, 2013: p. 1-29.

22. Bermudez, A. and Vazquez, M. E. (1994a). Upwind methods for hyperbolic conservation laws with source terms. Computers and Fluids, 23(8):1049-1071.

23. Kesserwani, G., Gerhard, N., Caviedes-Voullime, D., Haleem, D. A., and Muller, S. (2014). A multi-resolution discontinuous Galerkin method for one dimensional shallow water ow modelling. the 3rd IAHR Europe Congress with the theme Water Engineering and Research. Porto, Portugal.

24. Haleem, D. A., Kesserwani, G., and CaviedesVoullime, D. (2015). Haar waveletbased adaptive finite volume shallow water solver. Journal of Hydroinformatics. Vol. 17(6):857873

25. MacDonald, I. (1996). Analysis and computation of steady open channel flow. PhD. Thesis. University of Reading.

26. Burguete Tolosa, J., Garcia-Navarro, P., Murillo, J., et al. (2008). Friction term discretization and limitation to preserve stability and conservation in the $1 D$ shallow-water model: Application to unsteady irrigation and river flow. International Journal for Numerical Methods in Fluids, 58(4):403-425. 